\title{
Experimental investigation of elastomer mode I fracture: An attempt to estimate the critical strain energy release rate using SENT tests
}

\author{
David Roucou • Julie Diani - Mathias Brieu • Jean-François Witz • \\ Armel Mbiakop-Ngassa •
}

Received: date / Accepted: date

\begin{abstract}
The resistance to mode I failure of rubbers is studied by submitting single edge notch samples to uniaxial tension. Reproducing the seminal work of Rivlin and Thomas (1953), single edge notch tension specimens, presenting notches of various lengths, are stretched until break. A styrene butadiene rubber, unfilled and filled with carbon-black, and an unfilled rubber from the latter mentioned work, were considered. When the notch is smaller than one fifth of the sample width, mode I crack opening is observed, leading to catastrophic failure that creates smooth mirror-like crack surfaces. Nonetheless, the experimental force-elongation responses show that the mode I critical energy release rate cannot be calculated by a classical Griffith elastic failure analysis. When notches are longer, the SENT samples are not submitted to pure uniaxial tension only. Structural bending leads to uncontrolled mixed mode crack propagation. The surfaces created when the long notches propagate are rough and bifurcations are witnessed for the filled rubbers.
\end{abstract}

Keywords SENT tests - Strain energy release rate Mode I · Rubbers · Carbon-black

D. Roucou · M. Brieu · J.F. Witz

Laboratoire de mécanique de Lille, Ecole Centrale de Lille, Bd Paul Langevin, 59650 Villeneuve d'Ascq, France

E-mail: david.roucou@centrale.centralelille.fr

E-mail: mathias.brieu@centralelille.fr

E-mail: jean-francois.witz@centralellile.fr

J. Diani

LMS, Ecole Polytechnique, Route de Saclay, 91128 Palaiseau, France

E-mail: julie.diani@polytechnique.edu

A. Mbiakop-Ngassa

Manufacture Française des pneumatiques Michelin, CERL, Ladoux, 63040 Clermont-Ferrand, France

E-mail: armel.mbiakop-ngassa@michelin.com

\section{Introduction}

The resistance to failure of rubbers is a major issue for the rubber industry. Adding fillers to the rubber gum has proven to delay wear and crack propagation significantly. According to Rivlin and Thomas [14] seminal work, the energy release rate $G$ characterized on single edge notch specimens, may easily be determined by expression,

$G=2 K a \mathcal{U}$

with $a$ the length of the initial cut, $\mathcal{U}$ the stored elastic energy density, and $K$ a factor depending on the macroscopic stretch $\lambda$. Equation (1) was proposed based on geometrical and dimensional considerations using Griffith failure analysis [8]. The factor $K$ has been evaluated experimentally [7] and confirmed numerically [12] providing an expression of $K \simeq \frac{3}{\sqrt{\lambda}}$. Nonetheless, note that Yeoh [15] pointed out that the finite element analysis may result into a mere tautological argument. Eq. (1) has been reported in survey on fracture of rubbers [10, $13,2]$, and many authors ([11,9,6,5,3,4] among others) have applied it directly, conveniently bypassing the fastidious work of running Griffith analysis that demands to break several samples with increasing notch lengths, and calculating the critical energy release rate $G_{c}$ as the energy $G$ at which catastrophic failure is witnessed. Eq. (1) was established on an unfilled natural rubber, which raises the question of its extension to filled materials. Therefore, SENT tests have been carried out on a carbon-black filled styrene butadiene rubber (SBR). When results questioning the possible determination of the critical energy release rate for mode I fracture were obtained, additional tests were carried out on the unfilled SBR and finally on one of the unfilled natural rubbers tested by Rivlin and Thomas [14] which com- 


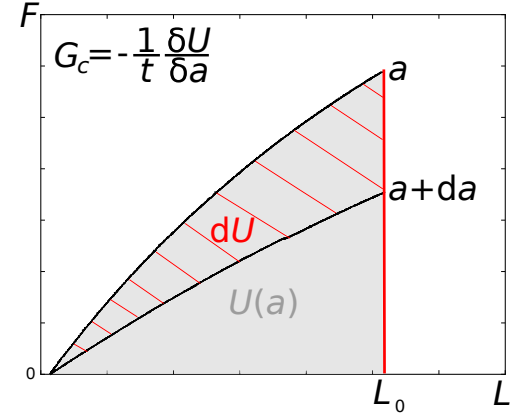

Fig. 1 Fragile fracture Griffith analysis at constant applied elongation.

position was found in their contribution. The obtained raw data are consistent with Rivlin and Thomas [14] plots, and show that when mode I fracture is unarguably witnessed the Griffith analysis leads to uncertain determination of $G$, questioning the use of Eq. (1).

\section{Theory}

In order to facilitate the reader's understanding, Rivlin and Thomas' experiments and analysis are reminded quickly. Applying Griffith analysis [8], one considers a specimen presenting a crack of length $a$, stretched until length $L_{0}$. The elongation is maintained constant while the crack propagates to reach length $a+d a$. The sample is then unloaded, and the elastic responses of the specimen for crack lengths $a$ and $a+d a$ are compared to calculate the critical energy release rate (Figure 1). For SENT specimens of constant thickness $t$, the critical energy release rate $G_{c}$ is defined as:

$G_{c}=-\frac{1}{t} \frac{\partial U}{\partial a}$

with $U$ the strain energy greyed out in Figure 1.

Rubbers show catastrophic failure, therefore it is not possible to stop the crack propagation at length $a+d a$ and unload the sample. For this purpose Rivlin and Thomas [14] proposed to submit several samples of various initial crack lengths to uniaxial tension and to compare their elastic responses. The propagation of the crack from $a$ to $a+d a$ is studied by comparing the forceelongation responses of samples with initial notches $a$ and $a+d a$. To apply Griffith theory, any other source of energy dissipation must be negligible during the crack propagation. When uniaxial tension is applied slowly enough, rubber viscoelasticity is negligible. Moreover, when studying unfilled natural rubbers, crystallization is likely to be very localized at the crack tip and therefore negligible [14]. Unlike unfilled rubbers, filled rubbers present nonlinear viscoelasticity at moderate strain
Table 1 Material mass compositions before vulcanization.

\begin{tabular}{llll}
\hline Ingredient & SBR50 & SBR0 & E \\
\hline SBR & 100 & 100 & \\
NR & & & 100 \\
N347 & 50 & & \\
Sulfur & 2.3 & 2.3 & 3 \\
6PPD & 1.9 & 1.9 & \\
Steraic acid & 2 & 2 & 0.5 \\
ZNO & 2.5 & 2.5 & 2 \\
CBS & 2.3 & 2.3 & \\
MBTS & & & 4 \\
\hline
\end{tabular}

(Payne effect) and Mullins softening that are sources of energy dissipation. Nonetheless, as long as one can show that the energies dissipated during the uniaxial tension in specimens of notch lengths $a$ and $a+d a$ are similar, Griffith analysis applies.

In what follows, specimens of various notch lengths are submitted to uniaxial tension and their force-elongation responses are compared to apply Griffith analysis like Rivlin and Thomas did.

\section{Materials and experiments}

\subsection{Materials}

All rubbers used in this study have been manufactured by Michelin. Three materials are considered, a $50 \mathrm{phr}$ carbon-black filled styrene butadiene rubber, the same unfilled rubber gum, and an unfilled natural rubber. The SBR gum was chosen for its non-crystallizing property. The star-branched solution SBR presents a molar mass of $M_{n}=120 \mathrm{~kg} / \mathrm{mol}$ with a styrene content of $15 \%$. Its entanglement density is close to $42.10^{-5}$ $\mathrm{mol} / \mathrm{cm}^{3}$. Fillers are N347 carbon-black. The natural rubber recipe was found in [14], where it is labelled $E$. Compositions of the materials are listed in table 1 . Materials were received as plates of approximately 2.4 $\mathrm{mm}$ thickness in which rectangular samples of $150 \mathrm{~mm}$ length and $30 \mathrm{~mm}$ width were cut.

\subsection{Experimental procedure}

A cut was hand-made with a razor blade on one of the long edges of each specimen, and its length was measured by microscope. The uniaxial tension tests were carried out on an Instron 5882 uniaxial testing machine, equipped with a $2 \mathrm{kN}$ load cell and pneumatic grips. Uniaxial tension was applied at constant crosshead speed. For each material, preliminary cycles have been run at $0.2,2$ and $20 \mathrm{~mm} / \mathrm{min}$ on different samples, to evaluate the effect of strain rate on the viscoelastic dissi- 
pation during a load-unload cycle, and define an optimal crosshead speed. As expected both unfilled rubbers show very little hystereses, unlike the filled SBR. More interestingly, for each material, the hystereses measured at $0.2 \mathrm{~mm} / \mathrm{min}$ and at $20 \mathrm{~mm} / \mathrm{min}$ are similar, varying of $3.4 \%$ only. Consequently, tests were run at 20 $\mathrm{mm} / \mathrm{min}$ in order to shorten the test duration. For the filled SBR every sample was pre-stretched up to $250 \%$ before making the notch in order to release most of Mullins softening.

For each material, the initial distance between the grips at zero force was set identical for every sample in order to mimic Rivlin and Thomas procedure easing the comparison between their results and ours. Upon uniaxial tension, load and crosshead displacements are recorded. For each sample, the elongation at break is marked by a full circle. The amount of work $W$ needed to deform a specimen up to length $L$ is defined by the area below the force-elongation curve up to $L$. Assuming reversible elastic deformation, this work is equal to the energy stored elastically in the rubber, i.e. $W=U$ . The quantity $W / t$ is then plotted with respect to the cut length $a$, in order to estimate $G_{c}$ according to Eq. (2). In addition, tests were videoed, providing images of the specimen upon stretching.

\section{Results and discussion}

\section{$4.150 \mathrm{phr}$ filled SBR fracture}

According to $[14,7]$, in order to ensure that the specimen is essentially in uniaxial tension, the initial length of the notch must be smaller than one fifth of the specimen width, which in the present study is $6 \mathrm{~mm}$. Several specimens with such small cuts were stretched until break. Their force-elongation responses are shown in Figure 2. Light color curves correspond to specimens of longer initial cuts, and the curves darken with specimens of smaller initial cuts. All force-elongation superimpose and show no dependence to the initial cut length. Consequently, when calculating $W / t$ for a given length $L$ with respect to the notch length $a$, one is unable to identify a trend. Figure 3 , where $W / t$ values were reported for $L=125 \mathrm{~mm}$ for instance, illustrates this result. Therefore, it is not possible to apply Eq. (2) and estimate an objective value for $G_{c}$. The errorbars in Figure 3 correspond to the experimental uncertainties estimated as follow. The cut lengths were evaluated as the average of two measures, one on each side of the specimen. The errorbars show the exact two measures. For $W / t$, a standard deviation of $1.6 \%$ was obtained by testing eight uncut samples and calculating the maxi-

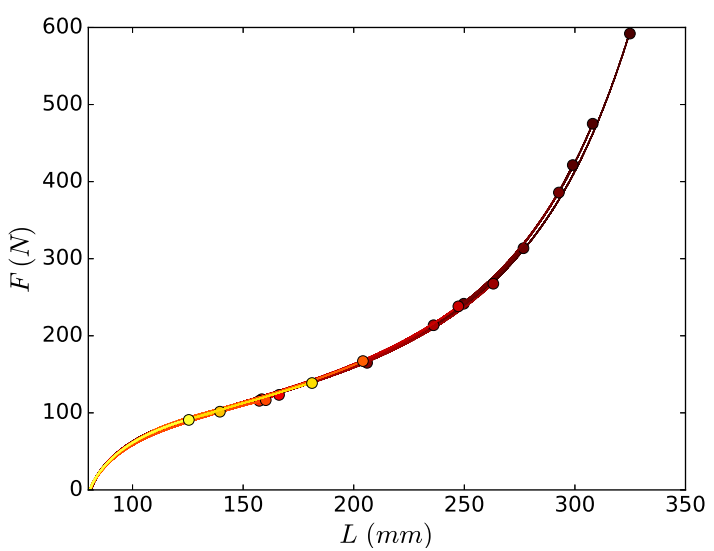

Fig. 2 Filled SBR force-sample length recorded during uniaxial tension for specimens with initial notches varying between $500 \mu \mathrm{m}$ to $6 \mathrm{~mm}$. The circles indicate the elongations at break. Light colors correspond to the longer notches.

mum standard deviation for $W / t$ at various given elongation $L$.

Very recently, Chen et al. [1] ran uniaxial tension tests on notched samples of acrylic elastomer and polyurethane. They showed that, when the notches are small enough, notched samples behave as unotched samples. They proposed to dismiss the Griffith elastic fracture analysis when the defects are small enough, and to use the work to rupture of uncut samples to predict specimen breaks. Unfortunately, their analysis that discusses rubber flaw sensitivity, does not apply to our results. Every specimen displayed fracture at the notch proving that the latter acts as a defect. Moreover, as shown in Figure 4, the stretch at break decreases with respect to the initial notch length even for the smaller notches, when Chen et al. [1] analysis is supported by a plateau of the stretch at break for the smaller notches.

At first sight, results showed in Figures 2 and 3 are different from Rivlin and Thomas [14] Figures 2 and 3 results. Actually, these authors have cut large razor notches on their SENT samples. As one can read in parts 4 and 5 in [14], the sample length, which is at least twice its width, is $12 \mathrm{~cm}$, while razor cuts vary between 1.17 and $5.7 \mathrm{~cm}$ (see Figure 2.(iii) and 4.(iii) in [14]). Therefore, long notches were also cut on the filled SBR samples in the present study. The force-elongation responses for SENT specimens with notches varying between 7.2 and $22.7 \mathrm{~mm}$ are shown in Figure 5 (keep in mind that the sample width is $30 \mathrm{~mm}$ ). The curves displayed in Figure 5 look very much like the curves presented in Figures 2 and 4 in [14]. When calculating $W / t$ with data shown in Figure 5, it is possible to estimate a value for $-\frac{1}{t} \partial W / \partial a$. 


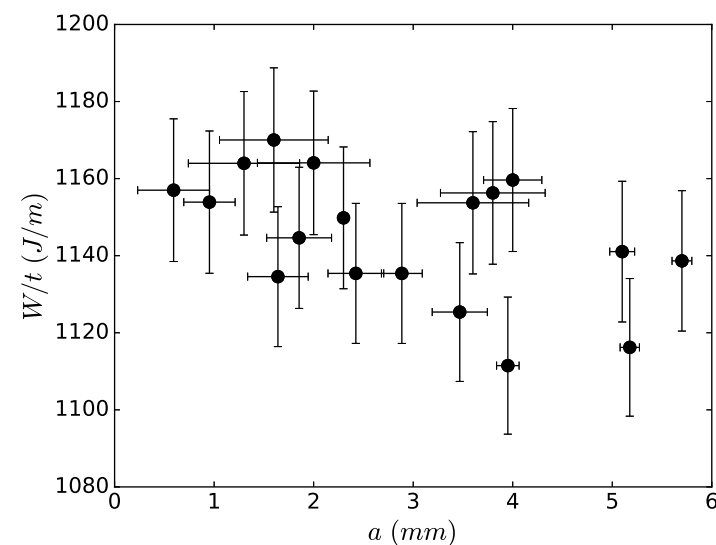

Fig. 3 Filled SBR values of $W / t$ with respect to initial cutlength calculated from figure 2 force-elongation responses at a chosen given length $L=125 \mathrm{~mm}$.

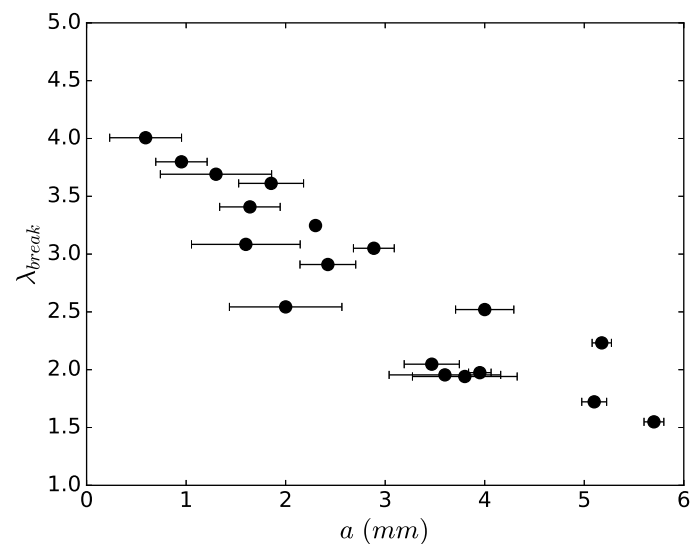

Fig. 4 Filled SBR stretch at break with respect to notch initial length extracted from figure 2 for small length notches.

The issue with large notches stands in the fact that the cut propagation is not happening in mode I. As shown in Figure 6 that presents images of the loaded sample before crack propagation for initial small (left) and large (right) notches. When the notch is initially small, the sample remains aligned with the direction of uniaxial tension, perpendicular to the white lines drawn. Consequently, except for the close vicinity of the crack, the sample is undergoing uniaxial tension strain. When the notch is initially large, the sample undergoes some rotation, highlighted by the rotation of the painted white lines initially perpendicular to the direction of tension, that changes its state of deformation mixing shearing and tension. Consequently, the crack is not submitted to pure mode I opening, and mixed mode propagation is rather observed as shows the crack path during propagation (Figure 7). When notches are small the crack propagates horizontally or equivalently per-

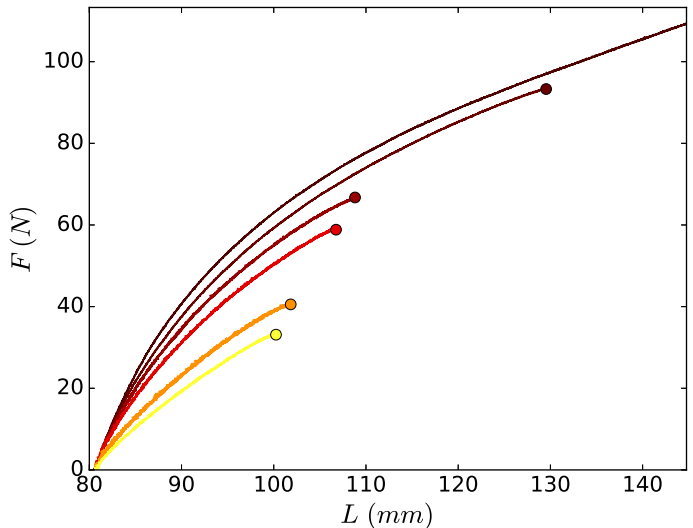

Fig. 5 Filled SBR force-sample length recorded during uniaxial tension for specimens with initial notch larger then 6 $\mathrm{mm}$. For reference, the top line shows the response of a specimen with a small notch. Lighter colors correspond to the longer initial notches. Circles mark the sample breaks.
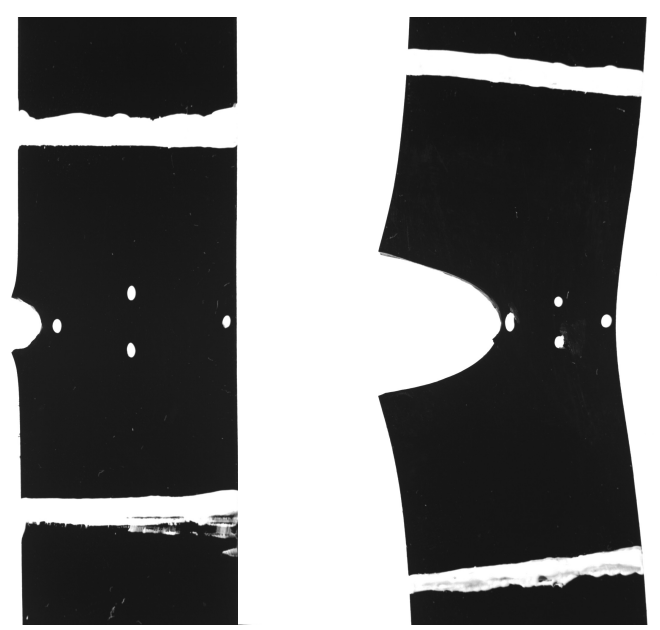

Fig. 6 Comparison of the SENT specimen states of deformation during applied uniaxial tension for a small notch (left) and a large notch (right) filled SBR samples.

pendicularly to the tension direction, when notches are long the crack bifurcates and propagates with an angle that varies between 45 and 60 degrees. Moreover, the examination of the surfaces created during the crack propagation shows that very smooth surfaces are obtained when the crack propagates horizontally only.

Therefore, it was observed on the tested filled SBR that when the notches propagate according to mode I opening, the Griffith macroscopic fracture analysis cannot be simply applied, discarding the use of Eq. (2) and questioning the use of Eq. (1). In order to appreciate the role of the carbon-black fillers in this result, the same experiments were run on the unfilled SBR gum. 


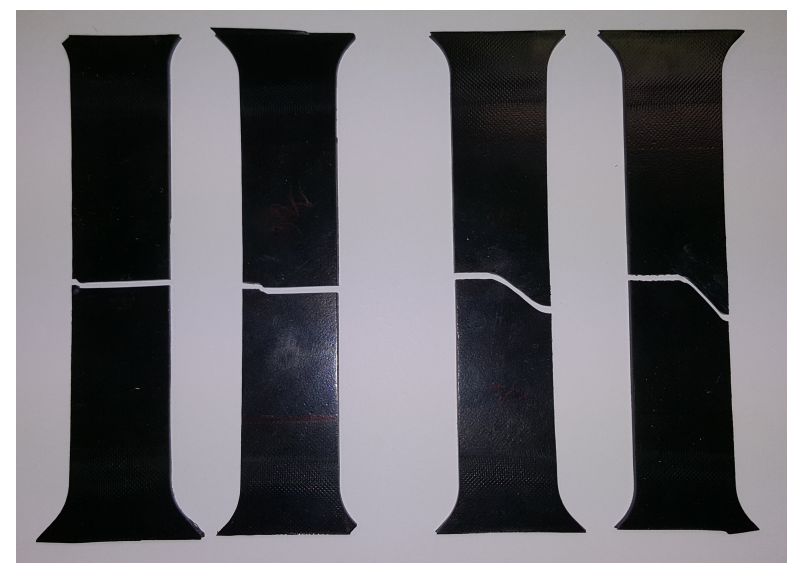

Fig. 7 Filled SBR crack paths according to their initial lengths: Horizontal propagation for small initial cuts and crack bifurcation for long initial cuts.

\subsection{Unfilled SBR mode I break}

Small razor cuts were made and the SENT samples were stretched until break. The crack propagation is unstable creating nice smooth surfaces (Figure 8). The specimen force-elongation responses are presented in Figure 9. As for the filled SBR, the force-elongation responses superimpose showing independence with respect to the initial cut length. Consequently, values of $W / t$ calculated up to a given displacement $L$ show an uncertain trend when plotted with respect to the notch length (Figure 11). As for the filled SBR, it is not possible to calculate a critical energy release rate when relatively small notches are opened. Figure 4.2 shows that the stretch at break decreases with the increasing notch length in a similar fashion to the filled rubber. When longer razor cuts were made, the SENT specimens show force-elongation responses declining with the increase of the notch length. As for the filled SBR, the samples rotate. Although the crack bifurcation was not witnessed for this material, the crack surfaces are rough resulting from mixed mode crack propagation unlike for small cuts which show smooth mirror surfaces (Figure 8). As a consequence, similar results and identical conclusions are obtained with the unfilled SBR. Finally, the natural rubber tested by Rivlin and Thomas is also studied.

\subsection{Failure of Rivlin and Thomas natural rubber $E$}

The same procedure was applied to the unfilled natural rubber $E$ following Rivlin and Thomas [14] formulation. Starting with notches varying between 1.5 and $5 \mathrm{~mm}$, one observes mode I horizontal unstable crack propagation. The force-elongation responses of the SENT samples upon stretching are plotted in Figure 12. As for

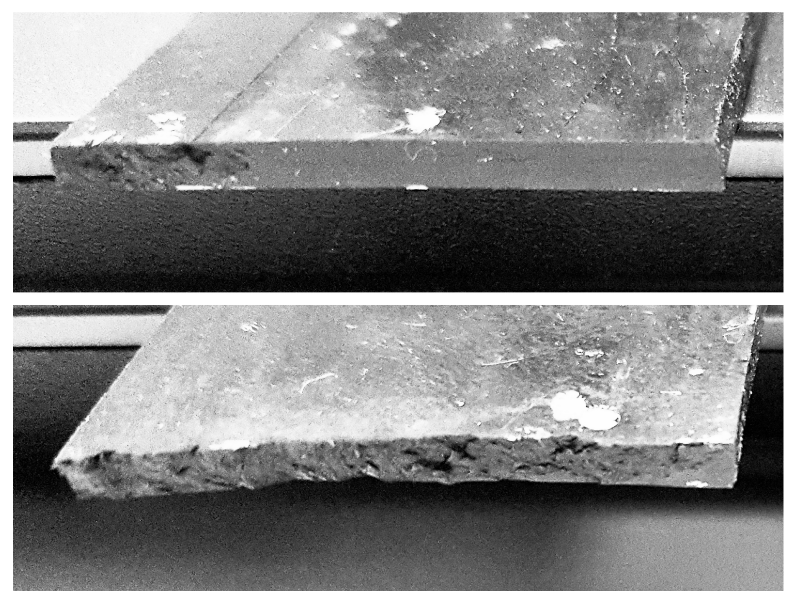

Fig. 8 Unfilled SBR surfaces created after crack propagations: smooth shiny surface for initial small cut (top) and rough surface for initial large notch (bottom).

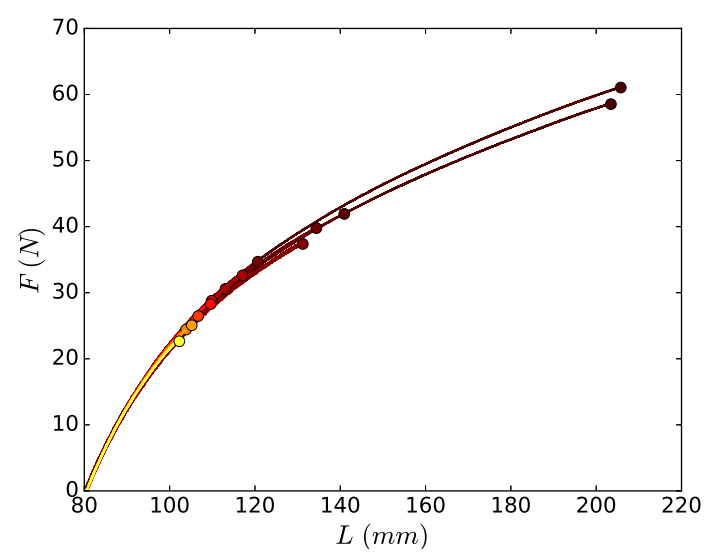

Fig. 9 Unfilled SBR force-sample length recorded during uniaxial tension for specimens with initial notches varying between $500 \mu \mathrm{m}$ to $5 \mathrm{~mm}$. The circles indicate the elongations at break. Light colors correspond to the longer notches.

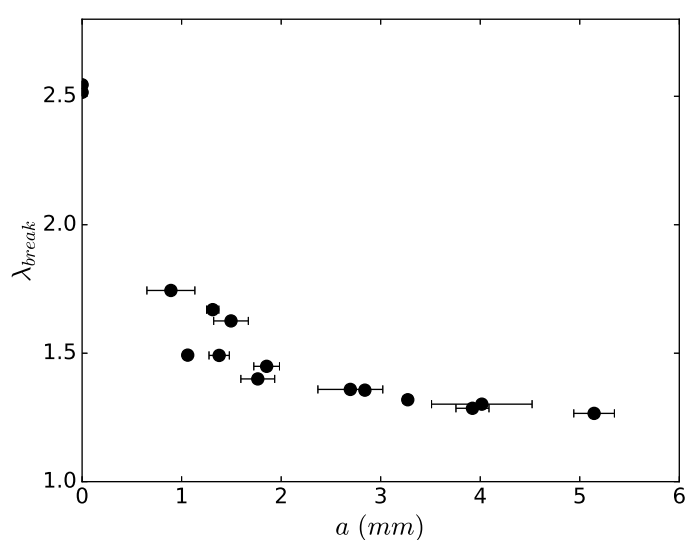

Fig. 10 Unfilled SBR stretch at break with respect to notch initial length extracted from figure 9 for small length notches. 


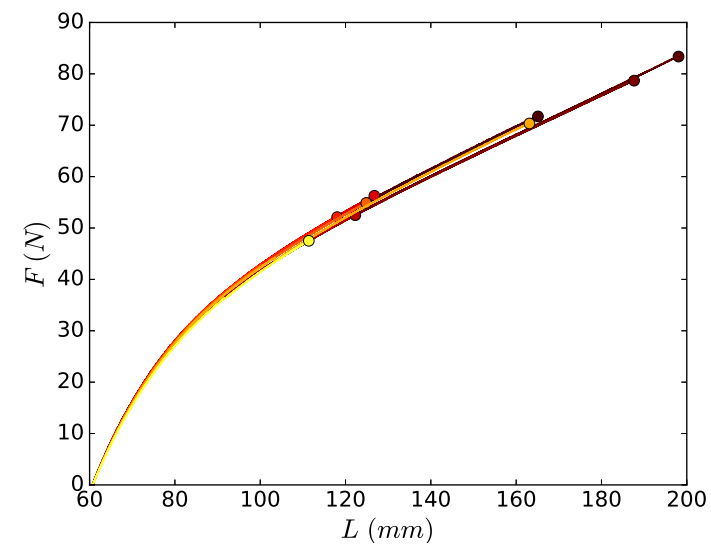

Fig. 11 Unfilled SBR values of $W / t$ with respect to initial cut-length calculated from figure 9 at a chosen given length $L=102 \mathrm{~mm}$.

the SBRs, the force-elongation responses superimpose, driving to values of $W / t$ at a given elongation $L$ that are independent of the initial notch length $a$ (Figure 13).

When increasing the notch length from 8.8 to 21 $\mathrm{mm}$, the sample rotates upon stretching (Figure 14), as highlighted by the curvature of the side opposed to the notch. Like for SBRs, the cracks is not submitted to pure mode I opening when the notch is initially long. Note that when long notches were considered, the crack could start propagating then stop, and propagate dramatically at larger elongation. The forceelongation responses become dependent on the initial cut length, decreasing as the cut length increases. This figures is in good agreement with Rivlin and Thomas force-elongation responses measured on SENT samples with long notches. Unlike Rivlin and Thomas, a value of $G_{c}$ was not calculated for the same reason as reported on filled SBR, the uncontrolled mixed mode propagation of the cracks.

\section{Conclusion}

Dealing with the characterization of fracture of rubbers and aiming at defining a fragile macroscopic criterion for mode I crack opening, SENT specimens were privileged. In an attempt to apply Griffith failure analysis, several samples of various initial hand-made razor notch lengths were stretched until break. It was observed that when the notch is reasonably small, smaller than one fifth of the specimen width, the sample is submitted to uniaxial tension favoring mode I crack propagation. The crack propagation is fragile creating nice smooth shiny surfaces. Nonetheless, the force-elongation responses su-

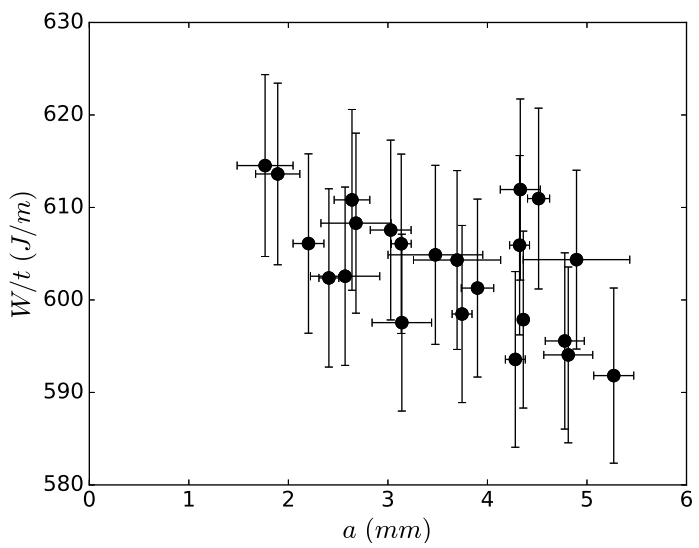

Fig. 12 Natural rubber force-sample length recorded during uniaxial tension for specimens with initial notches varying between $500 \mu \mathrm{m}$ and $5 \mathrm{~mm}$. The circles indicate the elongations at break. Light colors correspond to the longer notches.
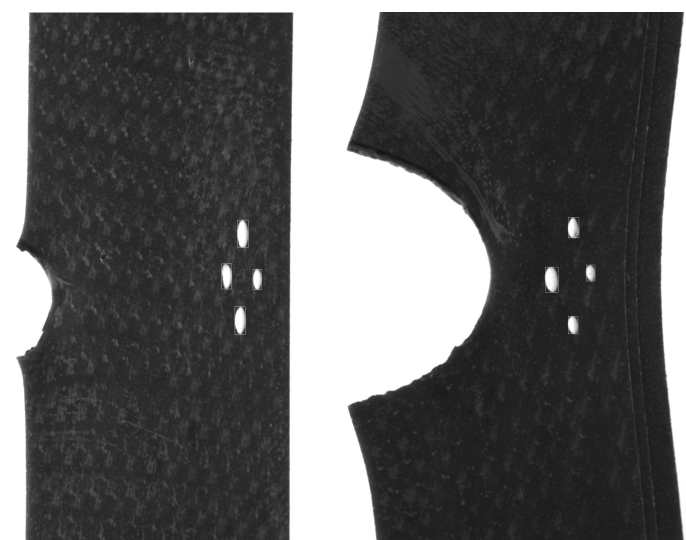

Fig. 13 Natural rubber values of $W / t$ with respect to initial cut-length calculated from figure 12 at a chosen given length $L=109 \mathrm{~mm}$.

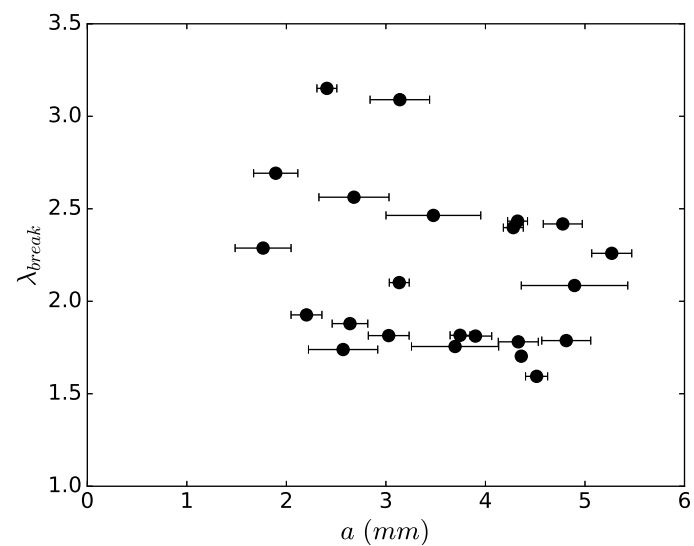

Fig. 14 Comparison of SENT specimen states of deformation during applied uniaxial tension for a small notch (left) and a large notch (right) natural rubber samples. 


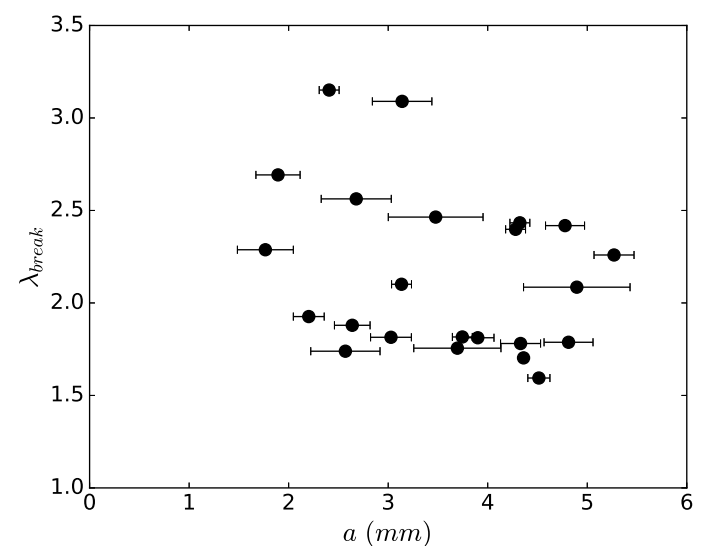

Fig. 15 Natural rubber stretch at break with respect to notch initial length for small length notches

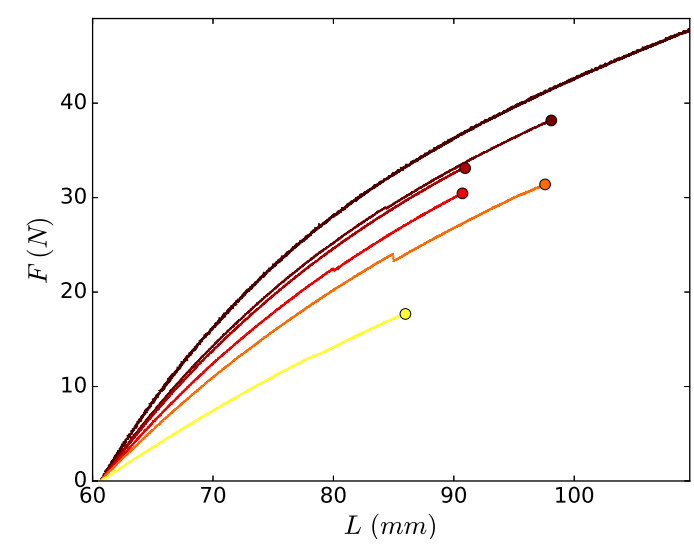

Fig. 16 Natural rubber force-sample length recorded during uniaxial tension for specimens with initial notch larger then 5 $\mathrm{mm}$. The top curve shows the behavior of a sample presenting a small notch. The circles indicate the elongations at break. Light colors correspond to the longer notches.

perimpose rendering impossible the determination of the strain energy release rate as defined by Griffith. Changes in the sample force-elongation responses necessary for the calculation of the strain energy release rate, were witnessed when notches are long only. In such cases, the samples were exhibiting states of strain different from uniaxial tension resulting in uncontrolled mixed mode crack propagation. The resulting rough crack surfaces or bifurcations confirm the mixed mode propagation.

The seminal results of Rivlin and Thomas [14] obtained on SENTS specimens, were established with large notches, ignoring the impact of mode mixity on crack propagation. Then, their results have been used by a large number of authors considering SENT specimens with small cuts and believing it could give access to mode I critical energy release rate. The present study strongly supports that more efforts should be done to prove that Eq. 1 applies and extends to every rubber. Next, two paths are envisioned, reproducing fracture tests on pure shear specimens and performing observations at a smaller scale in order to decide if an objective material fracture criterion may be extracted from classical tests.

\section{References}

1. Chen, C, Wang, Z, Suo, Z (2017) Flaw sensitivity of highly stretchable materials. Ext Mech Lett 10:50-57.

2. Creton, C, Ciccotti, M (2016) Fracture and adhesion of soft materials: a review. Rep Prog Phys 79:046601.

3. Cristiano, A, Marcellan, A, Keestra, BJ, Steeman, P, Creton, C (2011) Fracture of Model Polyurethane Elastomeric Networks. J Polym Phys Part B 49:355-367.

4. Diani, J, Brieu, M, Batzler, K, Zerlauth, P (2015) Effect of Mullins softening on mode I fracture of carbon-black filled rubbers. Int J Fract 194:11-18.

5. Gabrielle, B, Guy, L, Albouy, PA, Loc Vanel, L, Didier R. Long, D, Sotta P (2011) Effect of Tear Rotation on Ultimate Strength in Reinforced Natural Rubber. Macromolecules 44:7006-7015.

6. Gherib,S, Chazeau, L, Pelletier, JM, Satha H (2010) Influence of the Filler Type on the Rupture Behavior of Filled Elastomers. J Appl Polym Sci 118: 435-445.

7. Greensmith, HN (1963) Rupture of Rubber. X. The Change in Stored Energy on Making a Small Cut in a Test Piece Held in Simple tension. J Appl Polym Sci (1963)7:9931002.

8. Griffith, AA (1921) The phenomena of rupture and flow in solids. Philos Trans R Soc London A 221:163-198.

9. Hamed, GR, Park, BH (1999) The Mechanism of Carbon Black Reinforcement of SBR and NR Vulcanizates. Rubber Chem Technol 72:946-959.

10. Lake, GJ (1972) Mechanical fatigue of rubber. Rubber Chem Technol 45:309-328.

11. Lee, DJ, Donovan, JA (1985) Critical J-integral and tearing energies for fracture of reinforced natural rubber. Theor Appl Fract Mec 4:137-147.

12. Lindley, PB (1972) Energy for crack growth in model rubber components. J Strain Anal Eng Design 7:132-140.

13. Mars, WV, Fatemi A (2002) A literature survey on fatigue analysis approaches for rubber. Int J Fatigue 24:949961.

14. Rivlin, RS, Thomas, AG (1953) Rupture of rubber. I. Characteristic energy for tearing. J Polym Sci 10:291-318.

15. Yeoh, OH (2002) Relation between crack surface displacements and strain energy release rate in thin rubber sheets. Mech Mater 34:459:474. 